\title{
Periostin promotes chronic allergic inflammation in response to Th2 cytokines
}

\author{
Miho Masuoka, ${ }^{1}$ Hiroshi Shiraishi, ${ }^{1}$ Shoichiro Ohta, ${ }^{2}$ Shoichi Suzuki, ${ }^{1}$ Kazuhiko Arima, ${ }^{1}$ \\ Shigehisa Aoki, ${ }^{3}$ Shuji Toda, ${ }^{3}$ Naoki Inagaki, ${ }^{4}$ Yuichi Kurihara, ${ }^{5}$ Sayaka Hayashida, ${ }^{5}$ \\ Satoshi Takeuchi, ${ }^{5}$ Kenta Koike, ${ }^{6}$ Junya Ono, ${ }^{7}$ Hirokazu Noshiro, ${ }^{6}$ Masutaka Furue, ${ }^{5}$ \\ Simon J. Conway, ${ }^{8}$ Yutaka Narisawa, ${ }^{9}$ and Kenji Izuhara ${ }^{1,2}$
}

1Division of Medical Biochemistry, Department of Biomolecular Sciences, 2Department of Laboratory Medicine, and ${ }^{3}$ Department of Pathology and Biodefense, Saga Medical School, Saga, Japan. ${ }^{4}$ Department of Pharmacology, Gifu Pharmaceutical University, Gifu, Japan. ${ }^{5}$ Department of Dermatology, Kyushu University, Fukuoka, Japan. ${ }^{6}$ Department of Surgery, Saga Medical School, Saga, Japan. ${ }^{7}$ Shino-Test Corporation, Sagamihara, Japan. ${ }^{8}$ Developmental Biology and Neonatal Medicine Program, HB Wells Center for Pediatric Research, Indiana University School of Medicine, Indianapolis, Indiana, USA. ${ }^{9}$ Division of Dermatology, Department of Internal Medicine, Saga Medical School, Saga, Japan.

\begin{abstract}
Allergic inflammation triggered by exposure of an allergen frequently leads to the onset of chronic inflammatory diseases such as atopic dermatitis (AD) and bronchial asthma. The mechanisms underlying chronicity in allergic inflammation remain unresolved. Periostin, a recently characterized matricellular protein, interacts with several cell surface integrin molecules, providing signals for tissue development and remodeling. Here we show that periostin is a critical mediator for the amplification and persistence of allergic inflammation using a mouse model of skin inflammation. Th2 cytokines IL-4 and IL-13 stimulated fibroblasts to produce periostin, which interacted with $\alpha_{v}$ integrin, a functional periostin receptor on keratinocytes, inducing production of proinflammatory cytokines, which consequently accelerated Th2-type immune responses. Accordingly, inhibition of periostin or $\alpha_{v}$ integrin prevented the development or progression of allergen-induced skin inflammation. Thus, periostin sets up a vicious circle that links Th2-type immune responses to keratinocyte activation and plays a critical role in the amplification and chronicity of allergic skin inflammation.
\end{abstract}

\section{Introduction}

Inflammatory responses are triggered by insults on hosts, such as invasion of microbes or tissue damage, that the immune system senses and then eliminates or repairs (1). When a triggering insult is eliminated, the acute inflammatory responses are normally terminated, followed by transition to the homeostatic state, whereas failure to eliminate the inflammatory trigger (or some other reason) causes transition to a chronic inflammatory state. An allergen exposure can trigger allergic inflammation, leading to the onset of allergic diseases such as atopic dermatitis (AD) and bronchial asthma (2-4). The triggering allergens are recognized by an innate immune system consisting of DCs, basophils, or epithelial cells, followed by activation of acquired immunity in which Th2-type responses are dominant. It is generally accepted that persistent exposure to extrinsic allergens causes allergic inflammatory diseases to be chronic. However, there is no satisfactory explanation of why this should be so.

Fibrosis is a chronic pathological consequence of inflammatory responses (5). Often occurring as the end result of Th2-type responses, it leads to distortion of tissues and organs, sometimes impairing their functions (6). Fibrosis is characterized by de novo production and deposition of ECM proteins, such as collagens, fibronectin, elastin, proteoglycans, and laminin. Although maintenance of tissue structure is the main function of ECM proteins, some of them, such as osteopontin and tenascin-C, modulate immune responses by interacting with immune or nonimmune cells; these are termed matricellular proteins $(7,8)$.

Authorship note: Miho Masuoka and Hiroshi Shiraishi contributed equally to this work.

Conflict of interest: The authors have declared that no conflict of interest exists. Citation for this article: J Clin Invest. 2012;122(7):2590-2600. doi:10.1172/JCI58978.
Periostin (encoded by POSTN) is a recently characterized matricellular protein belonging to the fasciclin family $(9,10)$. Periostin interacts with several integrin molecules $-\alpha_{v} \beta_{1}, \alpha_{v} \beta_{3}$, and $\alpha_{v} \beta_{5}$ - on cell surfaces, providing signals for tissue development and remodeling. Analyses of Postn ${ }^{-/-}$mice have shown that periostin is important in the development of bone, tooth, and heart valves, as it acts on mesenchymal cells $(11,12)$. Periostin induces proliferation of cardiomyocytes, modulating the healing process of myocardial infarction $(13,14)$. We and others have recently shown that periostin has another physiological role in cutaneous wound repair (15-17). Furthermore, periostin is involved in the development of various tumors via the integrin/PI3K/Akt pathway (10). We previously found that periostin is a highly inducible product of IL-4 or IL-13, signature cytokines of Th2-type immune responses, in lung fibroblasts and is involved in fibrosis of bronchial asthma $(18,19)$, which suggested the involvement of periostin in allergic inflammation. Periostin enhances fibrosis via binding to other ECM proteins - collagen I, fibronectin, and tenascin-C - and collagen fibrillogenesis via activating lysyl oxidase (LOX), a catalytic enzyme for intra- and intermolecular cross-linking of collagen (19-21). Furthermore, several lines of evidence have suggested the importance of periostin as a matricellular protein in accelerating lung inflammation by enhancing chemokine production in fibroblasts, eosinophil recruitment, or TGF- $\beta$ activation in airway epithelial cells (22-24).

$\mathrm{AD}$ is a chronic, relapsing, highly pruritic inflammatory skin disease $(3,4)$. It is caused by the interaction of skin barrier damage and allergic skin inflammation in which the Th2-type immune response is dominant, particularly in the acute lesions. The pathogenesis of allergic skin inflammation in AD has been well characterized using various mouse models (4). The importance of Th2 cytokines, such as IL-4, IL-5, or IL-13, in allergic skin inflammation has been suggested by analyses based on genetically engi- 
neered mice and allergen- or hapten-treated mice. Furthermore, activation of keratinocytes is a hallmark of the pathogenesis of AD $(3,4)$. Activated keratinocytes in AD produce various proinflammatory cytokines and chemokines, among which thymic stromal lymphopoietin (TSLP), IL-25, and IL-33 are important in the initiation or amplification of Th2 responses $(2,25)$. In particular, TSLP has a critical role in initiation of the inflammatory cascade of AD at the interface between keratinocytes and DCs (26). However, it is not fully understood how Th2-type dominant inflammation and keratinocyte activation are linked in $\mathrm{AD}$.

In this study, we showed the involvement of periostin in chronic allergic inflammation using AD patients as well as a mouse model of allergic skin inflammation induced by epicutaneous sensitization with house dust mite extract (HDM). The requirement of periostin for the appearance of allergic skin inflammation was confirmed by its inhibition by genetic Postn deficiency or by blockage of its interaction with the receptor $\alpha_{v}$ integrin. We furthermore demonstrated, using a coculture system using keratinocytes and fibroblasts, that periostin produced by fibroblasts acted on keratinocytes, inducing production of proinflammatory cytokines - including TSLP - for Th2 skewing. These results suggest a unique role for periostin as an intrinsic mediator for amplifying and maintaining allergic skin inflammation by linking Th2-type inflammation and keratinocyte activation.

\section{Results}

Epicutaneous sensitization with HDM induces allergic skin inflammation in a Stat6-dependent manner in mice. To investigate the chronicity of allergic inflammation, we adopted a mouse model of allergic skin inflammation by epicutaneous sensitization with HDM, a common allergen associated with human AD (4). It has previously been reported that this mouse model exhibits phenotypes reminiscent of both acute and chronic AD lesions, including fibrosis, epidermal hyperplasia, spongiosis, lichenification, excoriation, and neurogenic inflammation $(27,28)$. We confirmed that mice epicutaneously sensitized with HDM exhibited ear swelling and redness, hyperplasia and dysregulated differentiation of the epidermis, and dermal fibrosis (Figure 1, A-C, and Supplemental Figure 1, A-D; supplemental material available online with this article; doi:10.1172/JCI58978DS1). Filaggrin, a related protein with skin barrier function, was prematurely expressed in the mid-spinous layer, as shown by staining that was broadened and reduced in intensity in HDM-treated WT mice, (Supplemental Figure 1C). This histological change was coincident with the impairment of skin barrier function, as observed in AD patients (29) and reported to be caused by IL-4 or IL-13 (30). The inflamed skin of these mice exhibited $\mathrm{CD}^{+} \mathrm{T}$ cell-dominant infiltration together with enhanced infiltration of eosinophils and mast cells (Supplemental Figure 2, A-D). Th2-type cytokines and chemokines were upregulated in ear skin lesions and draining lymph nodes (Figure 1D and Supplemental Figure 2E), although upregulation of IFN- $\gamma$, a clinical feature of chronic AD skin lesions $(3,4)$, was not observed (data not shown). Systemic responses to HDM (serum IgE and splenic T cell proliferation) were elevated as well (Supplemental Figure 2F and Supplemental Figure 3).

To examine whether allergic skin inflammation in HDM-sensitized mice depends on IL-4 and IL-13 signals, we applied epicutaneous HDM sensitization to mice deficient in Stat6, a common transcription factor of IL-4 and IL-13 signaling pathways (31). All morphologic changes, Th2-type inflammation, and skin barrier dysfunction induced by HDM sensitization disappeared in Stat $6^{-/-}$ mice (Figure 1 and Supplemental Figures 1 and 2). These results demonstrated that IL-4/IL-13 signals were critical for allergic skin inflammation in this mouse model.

Periostin is critical for induction of allergic skin inflammation in HDMsensitized mice. To gain insight into how IL-4/IL-13 signals can lead to allergic skin inflammation, we focused on periostin, an IL-4/ IL-13-inducible molecule $(18,19,32)$. We confirmed that IL-4 or IL-13 induced expression of periostin in both human and mouse dermal fibroblasts in vitro (Supplemental Figure 4). Induction of periostin was either little or not observed in other skin tissue cells, including keratinocytes (data not shown). Recurrent sensitization with HDM induced accumulation of periostin in the dermis of ear skin tissues, but this was not observed in Stat $6^{-/}$mice (Figure 1, $\mathrm{C}-\mathrm{E})$. Accumulation of periostin was observed in other mouse models of allergic skin inflammation (Supplemental Figure 5), using haptens (4-dinitrofluorobenzene [DNFB] and 2,4,6-trinitrochlorobenzene [TNCB]) or different mouse strains (C57BL/6 and $\mathrm{NC} / \mathrm{Nga}$ ), which strongly supports it is a common feature in mouse models of allergic skin inflammation. These results suggest that periostin is expressed in the skin tissues of HDM-sensitized mice downstream of IL-4/IL-13 signals.

To test the functional significance of periostin in the pathogenesis of allergic skin inflammation, we sought to induce allergic skin inflammation in Postn ${ }^{-/-}$mice with HDM. Periostin was expected to contribute to fibrosis formation by binding to other ECMs and inducing collagen formation in the chronic phase of allergic skin inflammation (19-21). Accordingly, in Postn ${ }^{-/-}$mice, both ear swelling and fibrosis induced by recurrent application of HDM were significantly suppressed (Figure 1, A and C, and Supplemental Figure 1D). Surprisingly, Postn deficiency caused impairment of Th2-type responses together with morphologic changes of epidermis, even with recurrent HDM stimuli (Figure 1D and Supplemental Figure 2). Downregulation of allergic skin inflammation was observed even in Postn ${ }^{-1-}$ mice on the C57BL/6 background $(\mathrm{H}$. Shiraishi, unpublished observations). These results suggest that periostin is a critical molecule of allergic skin inflammation.

Periostin enhances proliferation and differentiation of keratinocytes. Acanthosis - hyperproliferation and dysregulated differentiation of keratinocytes, a typical histological feature of $\operatorname{AD}(3,4)$ - was observed in the HDM-sensitized mouse model (Figure 1C). To examine the role of periostin in acanthosis, we reconstituted skin tissues in vitro with a 3-dimensional organotypic coculture system using keratinocytes and fibroblasts (Figure 2A and ref. 33). We used WT or Postn-/- fibroblasts in this system to examine the effects of periostin from fibroblasts on keratinocytes. We mimicked the AD skin lesions by adding IL-13 in the culture medium. Keratinocytes cultured with WT fibroblasts in the presence of IL-13 showed hyperplasia with increased numbers of PCNApositive cells, particularly in basal cells (Figure 2, B and C, and Supplemental Figure 6). These keratinocytes enhanced stratification and expression of CK14 and CK10, markers of basal and suprabasal layers, respectively, which excludes the possibility that epidermal hyperplasia is caused by impaired terminal differentiation. In contrast, Postn ${ }^{-/-}$fibroblasts did not induce proliferation or differentiation, even in the presence of IL-13, which indicates that periostin induced by IL-13 from fibroblasts acts on keratinocytes. Keratinocytes cocultured in periostin-deficient conditions showed an increase in TUNEL-positive cells (Figure 2C and Supplemental Figure 6). These data indicate that periostin enhances 

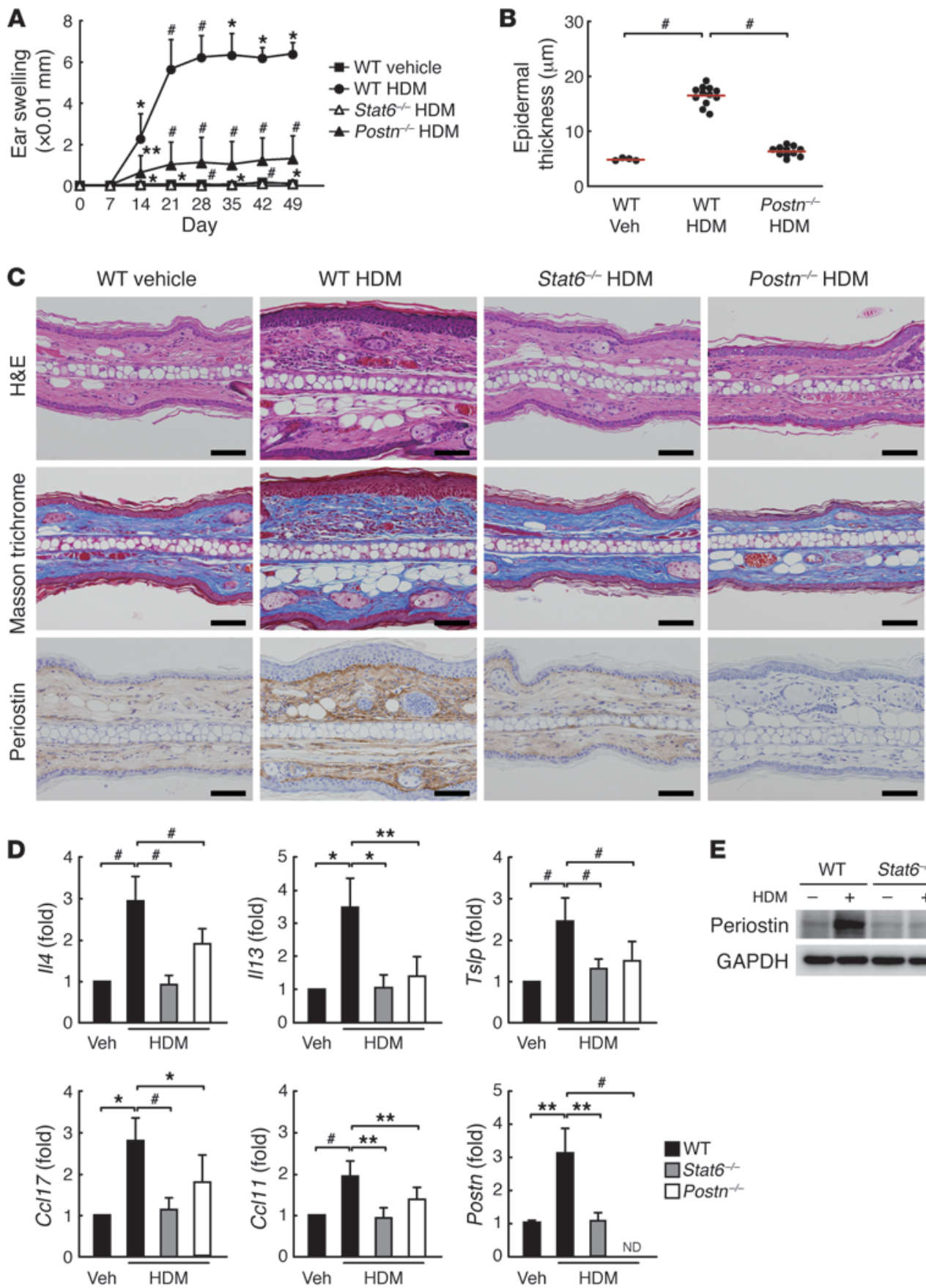

E

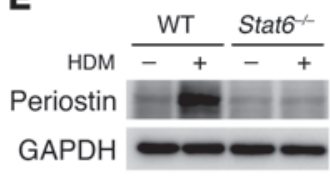

\section{Figure 1}

Allergic skin inflammation is STAT6/periostin dependent in the HDM mouse model. (A) Ear thickness of HDM-painted mice at the indicated times. $n=4$ (vehicletreated WT and HDM-painted Stat6 ${ }^{-/}$); 11 (HDM-painted WT and Postn ${ }^{-1-}$ ). (B-E) Mice were sacrificed at day 50 , and ear skin tissues were prepared. Experiments were done at least 3 times. (B) Epidermal thickness, measured in 10 views selected randomly. Red lines indicate means. (C) Staining with H\&E, Masson trichrome, and anti-periostin $\mathrm{Ab}$. Scale bars: $50 \mu \mathrm{m}$. (D) mRNA expression of the indicated cytokines, normalized to Actb. ND, not detected. (E) Western blot analysis of periostin and GAPDH. ${ }^{\star} P<0.05,{ }^{* \star} P<0.01,{ }^{\#} P<0.001$. the proliferation and survival of keratinocytes. Accordingly, we found abundant phosphorylation of Akt, which is important for survival, proliferation, and differentiation of keratinocytes (34), in the suprabasal keratinocytes cultured in the presence of periostin (Figure 2C and Supplemental Figure 6). Thus, periostin enhanced proliferation and differentiation of keratinocytes, which suggests a crucial role of periostin in acanthosis.

Activation of keratinocytes by periostin enhances Th2 inflammation. We then examined the effects of periostin on expression of keratinocyte-derived cytokines known to be important in skin inflammation: TSLP, TNF- $\alpha$, GM-CSF, IL-1 $\alpha$, IL-25, and IL-33 (2-4, 25). Coculture of keratinocytes and control fibroblasts in the presence of IL-13 induced expression of TSLP, TNF- $\alpha$, GM-CSF, and IL- $1 \alpha$, but not IL-25 or IL-33, whereas their production was markedly suppressed by Postn ${ }^{-/-}$fibroblasts (Figure 2D and data not shown). We assumed that inflammatory cytokines and chemokines derived from keratinocytes act on various immune cells, including APCs. We analyzed the effects of the conditioned medium of keratinocytes cocultured with fibroblasts (KCM) on DC function as a representative of the interaction between these cytokines/chemokines and immune cells, because TSLP, TNF- $\alpha$, GM-CSF, and IL- $1 \alpha$ activate or differentiate DCs to induce Th2 skewing $(26,35,36)$. We tested whether KCM induced Th2 cells via activation of DCs (Figure 2E). DCs stimulated by IL-13-treated KCM induced more 
A

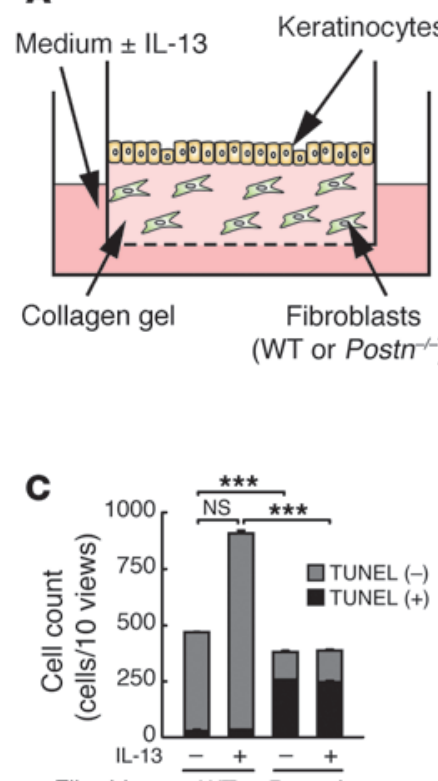

Fibroblasts $\overline{\text { WT }} \overline{\text { Postn }^{-1}}$
B
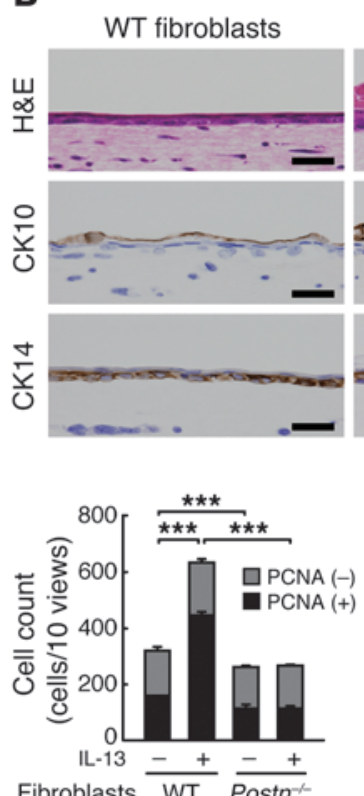

Fibroblasts $\frac{}{\text { WT }} \frac{+}{\text { Postn }}$
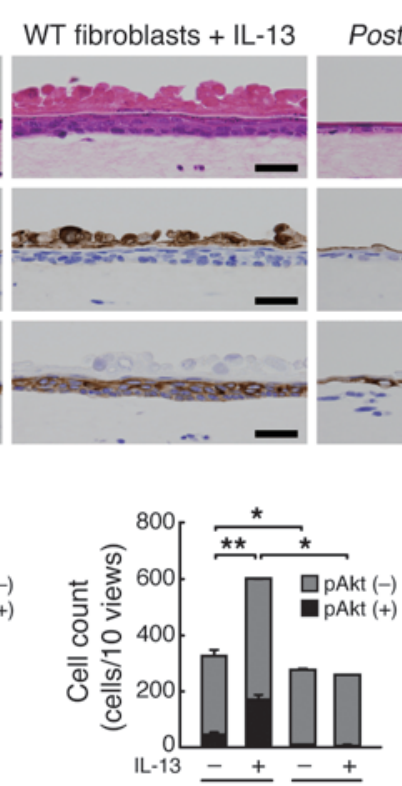

Fibroblasts $\overline{\text { WT }} \overline{\text { Postn }}$
D

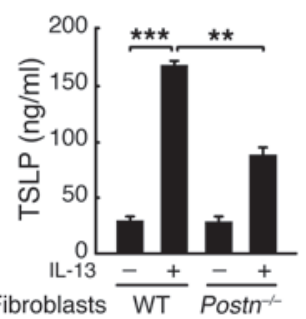

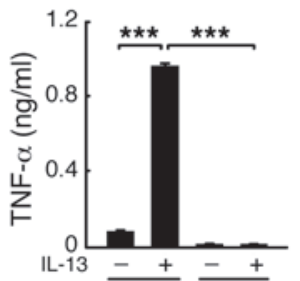

Fibroblasts $\overline{\text { WT }} \overline{\text { Postn }}$

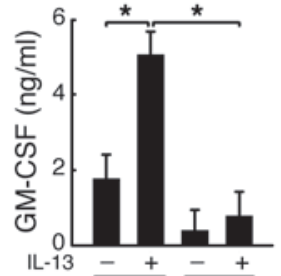

Fibroblasts $\overline{\text { WT }} \overline{\text { Postn }}$

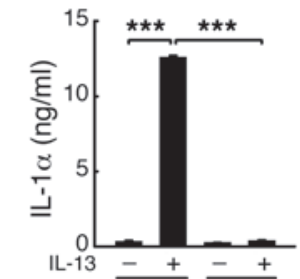

Fibroblasts $\overline{\text { WT }} \overline{\text { Postn }}$

E

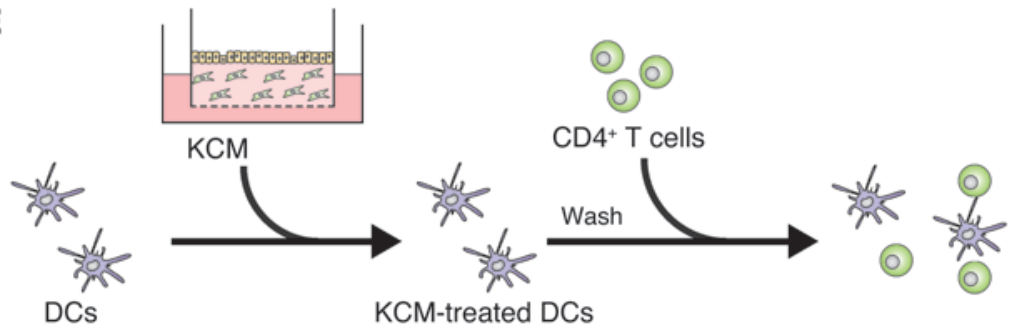

$\mathbf{F}$

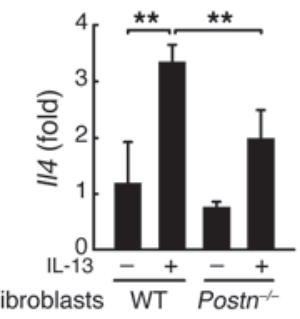

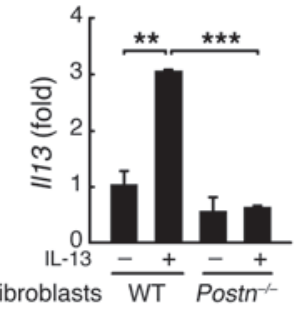

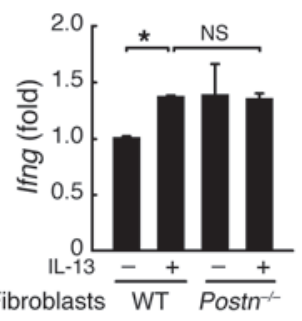

Postn ${ }^{-}$- fibroblasts

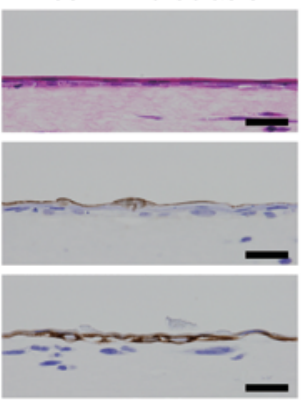

Postr ${ }^{-1}$ fibroblasts

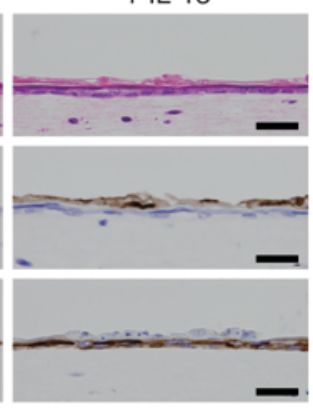

Figure 2

Periostin-induced proliferation, differentiation, and activation of keratinocytes in the 3-dimensional organotypic coculture system. (A) 3-dimensional organotypic coculture system. Keratinocytes were cocultured with Postn-1- or WT mouse-derived fibroblasts in the presence or absence of $10 \mathrm{ng} / \mathrm{ml}$ IL-13. (B) Histological staining with H\&E, anti-CK10, and anti-CK14 of the keratinocytes cultured for 7 days. Scale bars: $25 \mu \mathrm{m}$. (C) Number of cells positive or negative for TUNEL, PCNA, and phospho-Akt in histological staining, counted in $10 \times 200$ views per section. (D) Amount of TSLP, TNF- $\alpha$, GM-CSF, and IL-1 $\alpha$ in the supernatant in keratinocytes from day 5 to day 7. (E) Mixed lymphoid reaction system. Allogeneic CD4+ T cells were cocultured with bone marrow-derived DCs treated with the supernatants from the culture as in $\mathbf{A}$ for 5 days. (F) Quantitative RT-PCR analysis for II4, II13, Ifng, and II17a in mRNA samples prepared from allogeneic CD4+ T cells. Experiments were done at least 3 times. ${ }^{*} P<0.05$, ${ }^{* \star} P<0.01,{ }^{* * *} P<0.001$. 

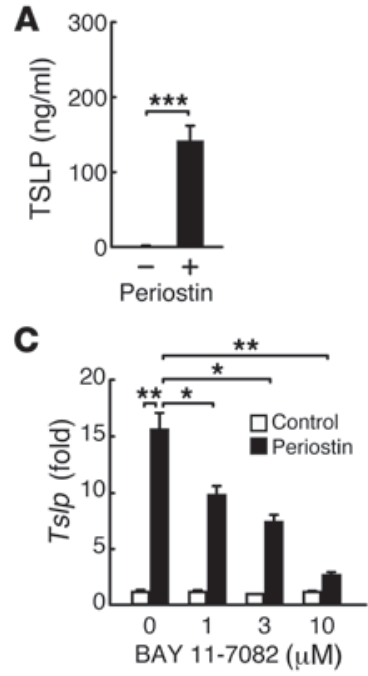
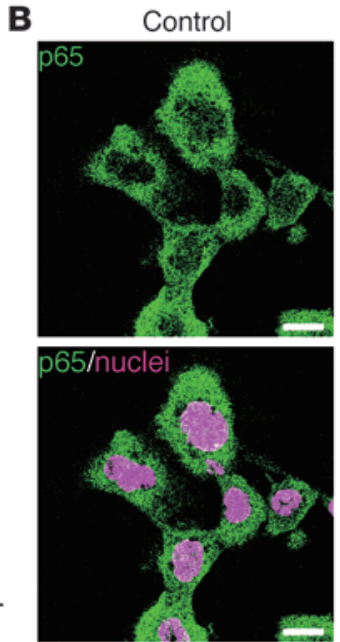

Periostin

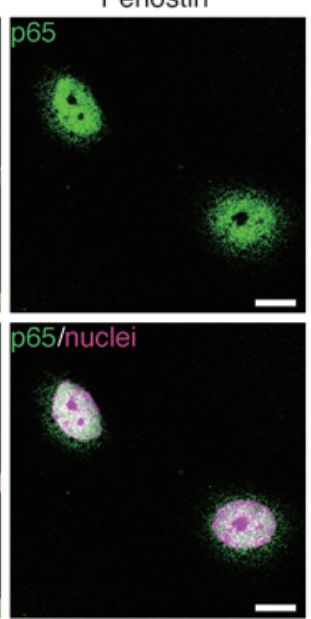

\section{Figure 3}

Induction of TSLP production by periostin through the NF-кB pathway in keratinocytes. Keratinocytes were cultured on precoated periostin for 72 (A) or 24 (B and C) hours. (A) TSLP production in the culture medium. (B) Confocal microscopic imaging of keratinocytes. Green and magenta represent p65 and nucleus, respectively. Scale bars: $10 \mu \mathrm{m}$. (C) Expression of Ts/p mRNA with the indicated concentrations of the NF-KB inhibitor BAY 11-7082. Experiments were done at least 3 times. ${ }^{\star} P<0.05,{ }^{* \star} P<0.01,{ }^{* * *} P<0.001$. proliferation of $\mathrm{CD}^{+} \mathrm{T}$ cells and augmented expression of IL-13 and IL-4, but not IFN- $\gamma$, compared with untreated KCM (Figure 2F and Supplemental Figure 7). Th2 skewing by IL-13-treated KCM was partially inhibited by addition of neutralizing Abs against TSLP (Supplemental Figure 8), suggestive of TSLP involvement in this event. Interestingly, IL-17A, a signature cytokine of Th17, was also induced in this condition. However, DCs stimulated by IL-13-treated KCM of Postn ${ }^{-/-}$fibroblasts impaired the proliferation of T cells and the induction of IL-13, IL-4, and IL-17A from $\mathrm{T}$ cells. Indeed, the migration of Langerhans cells and dermal DCs to draining lymph nodes and the expression level of MHC class II in skin DCs were reduced in Postn ${ }^{-/-}$versus littermate WT mice (Supplemental Figure 9). These results demonstrated that periostin enhanced Th2 skewing through activation of keratinocytes.

Periostin directly induces TSLP production via NF- $\mathrm{B} B$ activation in keratinocytes. We then examined the direct effects of periostin on production of TSLP, a key molecule for initiation of the cascade in allergic skin inflammation (26), in keratinocytes. When cultured on periostin-coated plates, keratinocytes produced significant amounts of TSLP, an effect that was enhanced by the addition of IL-13 (Figure 3A and data not shown). Interestingly, we did not observe the significant effect of periostin for proliferation of keratinocytes in monolayered culture condition (data not shown). It

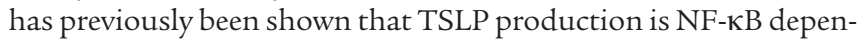
dent (26), which raises the possibility that periostin activates the NF-KB pathway in keratinocytes, followed by production of TSLP. Keratinocytes cultured on periostin-coated plates displayed the nuclear translocation of p65 (Figure 3B). Furthermore, BAY

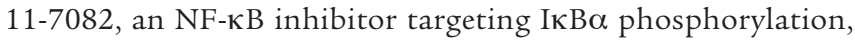
inhibited periostin-induced TSLP expression in a dose-dependent manner in keratinocytes (Figure 3C). These results demonstrated that direct interaction of periostin with keratinocytes induced

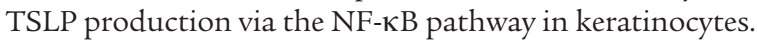

Interaction of periostin with $\alpha_{v}$ integrin is critical for the appearance of allergic skin inflammation. Periostin acts as a matricellular protein by binding to several integrin molecules - such as $\alpha_{v} \beta_{1}, \alpha_{v} \beta_{3}$, and $\alpha_{v} \beta_{5}$ - on cardiocytes, endothelial cells, and malignant cells, which enhances these cells' proliferation $(9,10,37)$. Among these integrins, $\alpha_{v} \beta_{3}$ was expressed predominantly on the suprabasal keratinocytes in the epidermis of normal donors and more broadly in
AD patients (Supplemental Figure 10). This finding, taken together with the importance of fibroblast-derived periostin for keratinocyte activation shown by the 3-dimensional organotypic coculture system, strongly suggests that the interaction of periostin with $\alpha_{v}$ integrin on cell surface evokes allergic skin inflammation. In the 3-dimensional organotypic coculture system and the monolayered culture system, hyperproliferation, dysregulated differentiation, and production of proinflammatory cytokines in keratinocytes were completely inhibited by neutralizing Abs against $\alpha_{v}$ integrin, and to a lesser extent by neutralizing Abs against $\beta_{3}$ integrin (Figure 4, A and B, and Supplemental Figure 11). To examine whether binding of periostin to integrin is important for allergic skin inflammation in HDM-sensitized mice, we blocked integrin signals by subcutaneously administering neutralizing anti- $\alpha_{v}$ integrin Abs concomitantly with HDM sensitization; the phenotypic changes induced by HDM were completely suppressed, similar to the observations in Stat6 ${ }^{-/-}$and Postn ${ }^{-/-}$mice (Figure 4, C-F, and Supplemental Figure 12). Furthermore, administration of neutralizing anti- $\alpha_{v}$ integrin Abs was effective even in established $\mathrm{AD}$, stopping and improving the progression of skin inflammation, including ear thickness, infiltration of inflammatory cells, epidermal thickness, and IgE production (Figure 5). These results support the notion that the interaction between periostin and $\alpha_{v}$ integrin is critical for generation of allergic skin inflammation in HDM-sensitized mice.

Periostin is highly expressed in the skin tissues of $A D$ patients and is correlated with disease severity. Finally, we examined whether the findings obtained from HDM-sensitized mice and in vitro experiments reflected the pathogenesis of AD patients. Expression of periostin was observed only slightly in the dermis on the border of the epidermis in normal skin tissues $(n=4)$, whereas all AD samples investigated $(n=27)$ and the HDM-sensitized mice showed significantly elevated expressions of periostin in the dermis, with a range of expression levels (Figure 6A). We grouped periostin expression levels into mild $(n=3)$, moderate $(n=11)$, and marked $(n=13)$. Inflammation severity, acute change (lymphocyte infiltration in dermis), and chronic changes (epidermal thickness and eosinophil infiltration in dermis) were well correlated with periostin expression levels (Figure 6B and Supplemental Figure 13). Furthermore, serum levels of periostin were significantly elevated in $\mathrm{AD}$ patients 
A
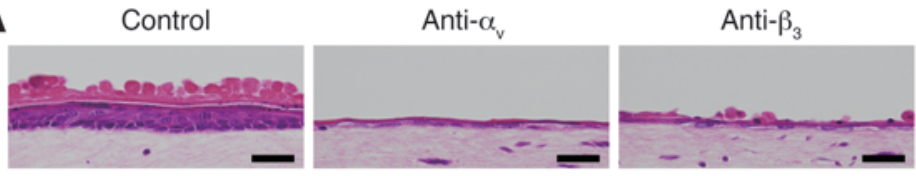

Anti- $\alpha_{\mathrm{v}}$ and anti- $\beta_{3}$

B

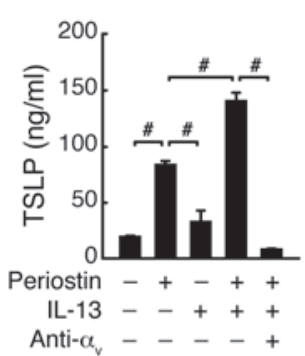

C

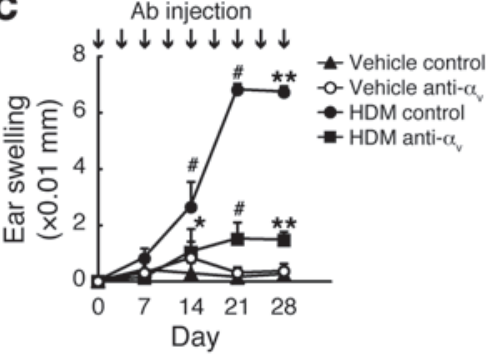

D

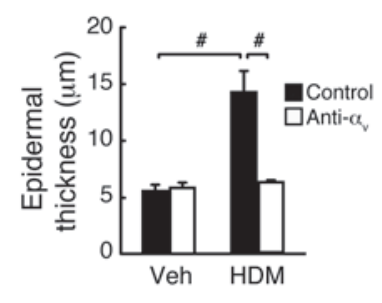

E

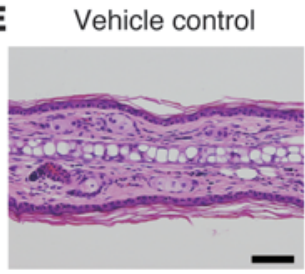

Vehicle anti- $\alpha$

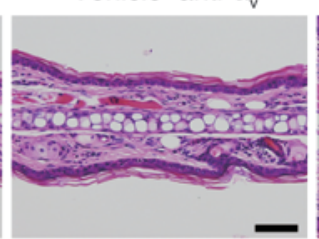

HDM control

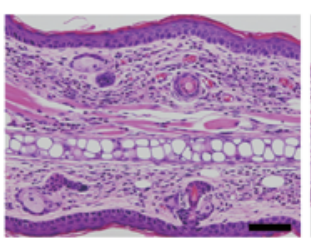

HDM anti- $\alpha_{v}$

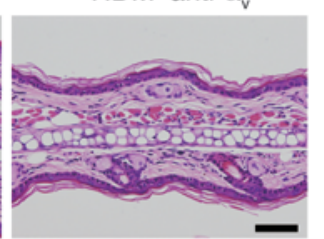

$\mathbf{F}$
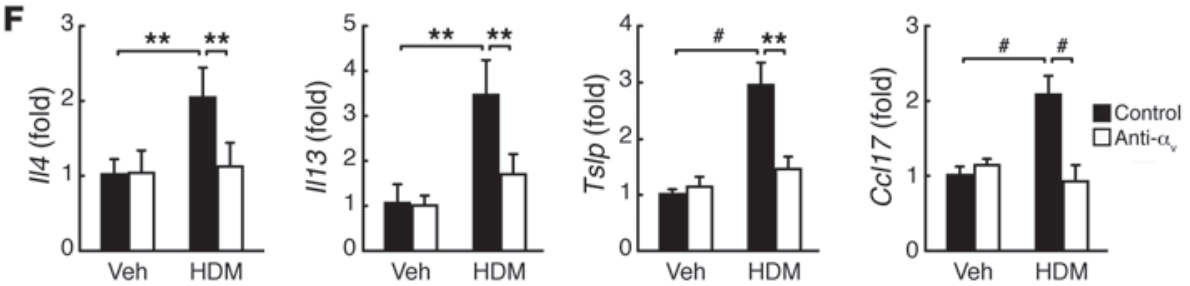

\section{Figure 4}

Requirement of the integrin/periostin interaction on keratinocytes for generation of allergic skin inflammation. (A) H\&E staining of keratinocytes cocultured with WT mousederived fibroblasts $\left(2 \times 10^{5}\right.$ cells) in the presence of $10 \mathrm{ng} / \mathrm{ml} \mathrm{IL-13}$ and control, anti- $\alpha_{v}$ integrin, and/or anti$\beta_{3}$ integrin Abs in the 3-dimensional organotypic coculture system. Scale bars: $25 \mu \mathrm{m}$. (B) TSLP production in keratinocytes cultured on a dish coated with $10 \mu \mathrm{g} / \mathrm{ml}$ recombinant periostin in the presence of IL-13 and anti- $\alpha_{v}$ integrin $\mathrm{Ab}$. Experiments were done at least 3 times. (C-F) Preventive protocol. BALB/c mice were treated with vehicle ( $n=3$ per group) or HDM ( $n=5$ per group) once per week for 4 weeks and injected subcutaneously with control or anti- $\alpha_{v}$ integrin $A b$ twice per week, then examined on day 29. (C) Ear swelling. (D) Epidermal thickness. (E) Skin histology with H\&E. Scale bars: $50 \mu \mathrm{m}$. (F) mRNA expression of the indicated cytokines. ${ }^{\star} P<0.05,{ }^{\star \star} P<0.01$, ${ }^{\#} P<0.001$. $(n=29)$ compared with healthy volunteers $(n=66$, Figure 6C). These results provided strong evidence in support of the involvement of periostin in $\mathrm{AD}$ pathogenesis.

\section{Discussion}

$\mathrm{AD}$ is a complex disease caused by the interaction of skin barrier damage and allergic skin inflammation $(3,4)$. It is well known that Th2-type inflammation is dominant in allergic skin inflammation in $\mathrm{AD}$, based on analyses of $\mathrm{AD}$ patients and the HDM-sensitized mouse model. Furthermore, activation of keratinocytes producing various proinflammatory cytokines and chemokines contribute to the initiation or amplification of Th2 responses $(2,25)$. However, it remained unclear how Th2-type inflammation and activation of keratinocytes interact, leading to amplification and chronicity of AD inflammation. In this study, we demonstrated that periostin, a recently characterized matricellular protein, represents a key mediator for amplification and chronicity of allergic skin inflammation, as it linked Th2 responses and keratinocyte activation.

Deficiency of Postn or inhibition of $\alpha_{v}$ integrin prevented development and/or progression of HDM-induced skin inflammation in our mouse model (Figures 1, 4, and 5 and Supplemental Figures 1,2 , and 12). We confirmed that Postn ${ }^{-1-}$ mice did not have endogenous hematopoietic or immune component defects and showed normal distribution and function of dermal DCs and Langerhans cells (Supplemental Table 1, Supplemental Figure 14, and ref. 11), excluding the possibility that primary immunodeficiency causes unresponsiveness to the allergen treatment in Postn ${ }^{-/-}$mice. Th2-type inflammation initiated by allergen exposure induced production of periostin in fibroblasts through IL-4 and IL-13. Accumulated periostin directly acted on keratinocytes to produce proinflammatory cytokines, including TSLP, via $\alpha_{v}$ integrin. The proinflammatory cytokines from keratinocytes then amplified the Th2 immune response. Considering all results from this study, we propose a model in which accumulation of periostin constitutes a pathogenic vicious circle that amplifies Th2-type inflammation by binding to $\alpha_{v}$ integrin on keratinocytes (Figure 7). This model suggests that amplification of allergic skin inflammation requires 2 pathways: exposure of APCs, including DCs, to allergens; and enhancement of immune responses, including activation of APCs, by keratinocyte-derived proinflammatory cytokines induced by periostin. The impaired induction of allergic skin inflammation observed in HDM-treated Postn ${ }^{-/-}$mice (Figure 1) indicates that even recurrent stimulation by allergens is not enough to establish allergic skin inflammation without the latter pathway. These effects may be essential in order for APCs to respond efficiently to exogenous allergens in allergic skin inflammation.

The roles of matricellular proteins in modulating immune and nonimmune cells in inflamed tissues have been recently elucidated. An intracellular isoform of osteopontin in DCs repressed Th17 cell differentiation in a type I, IFN receptor-dependent manner (8). Tenascin-C induces proinflammatory cytokines via TLR4 in macrophages and fibroblasts derived from rheumatoid 
A
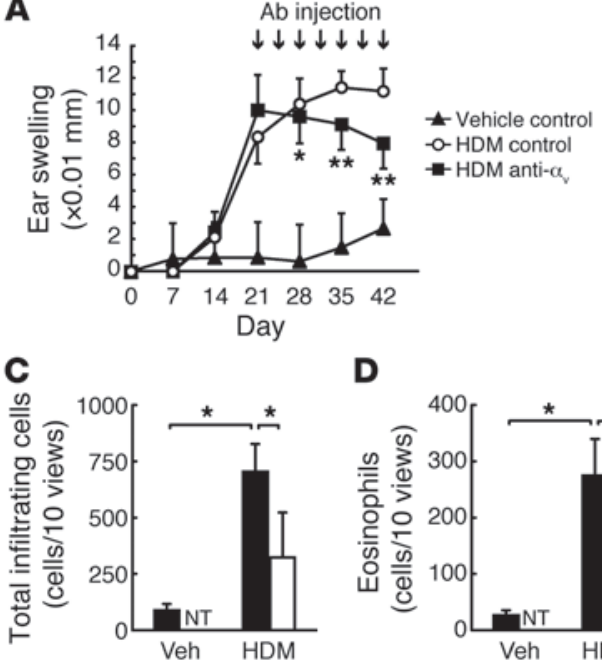

D

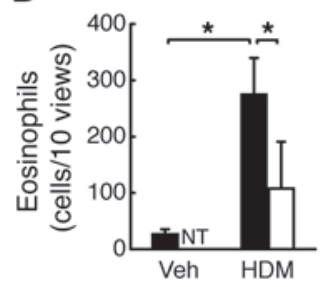

B Vehicle control

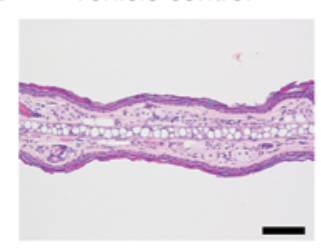

E

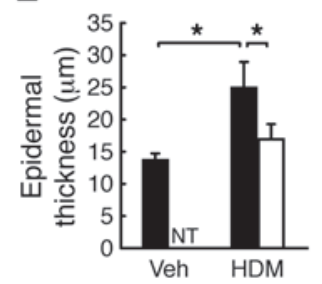

HDM control

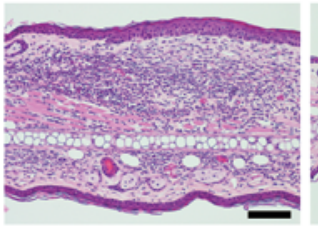

HDM anti- $\alpha$

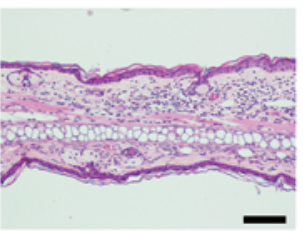

F

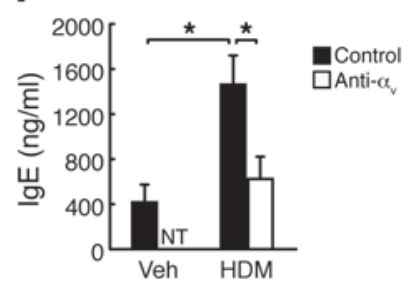

\section{Figure 5}

Blockage of the integrin/periostin interaction is effective in the mouse model of established skin inflammation. (A-F) Therapeutic protocol. WT mice treated with vehicle ( $n=4$ per group) or $\operatorname{HDM~(~} n=6$ per group) were administered control or anti- $\alpha_{v}$ integrin Ab by local injection twice weekly for 3 weeks from day 21. Mice were harvested 24 hours after the final painting of HDM (day 43). (A) Ear swelling. (B) Skin histology with H\&E. Scale bars: $100 \mu \mathrm{m}$. (C) Total number of infiltrated cells. (D) Number of infiltrated eosinophils. (E) Epidermal thickness. (F) Serum IgE. NT, not tested. ${ }^{\star} P<0.05,{ }^{\star *} P<0.01$.

arthritis patients (7). In this study, we demonstrated the significance of periostin on keratinocytes; however, we cannot exclude the possibility that periostin directly acts on immune cells or nonimmune cells other than keratinocytes, modulating their functions. Actually, it has been shown that periostin acts on eosinophils, enhancing their transmigration, chemotaxis, and adhesion (23). Furthermore, periostin activates TGF- $\beta$ via epithelial cells, followed by induction of collagen production (24). Immune cells and parenchymal/stromal cells activated by periostin together could orchestrate $\mathrm{AD}$ inflammation.

Several papers have recently shown the role of periostin in allergic airway inflammation. Sehra et al. and Gordon et al. showed that periostin protects mice from allergic airway inflammation $(38,39)$, whereas Blanchard et al. have shown that periostin accelerates allergen-induced eosinophil recruitment in lung and esophagus (23). Almost the same protocol using intranasal administration of Aspergillus fumigatus caused the different outcomes (23, 39 ), which suggests that the role of periostin in allergic airway inflammation remains unclear. We here established a persistent Th2-type skin inflammation model, and the outcomes using Postn ${ }^{-/}$mice were different from those of the airway model. This may be explained by the apparent persistence of Th2-type inflammation in the skin model, in contrast to the airway model, which is generally self-limiting in the mouse and resolves spontaneously over time. These results suggest that the skin model is more relevant than the airway model to analyze the chronicity of Th2-type inflammation and that the different contribution of periostin to chronic or acute allergic inflammation may explain the different outcomes in the skin and airway models.

$\mathrm{HDM}$ is a common allergen associated with human $\mathrm{AD}$ and bronchial asthma $(4,40)$. In the present study, we demonstrated that HDM-treated mice exhibited phenotypes reminiscent of both acute and chronic AD lesions (Figure 1 and Supplemental Figures 1 and 2$)$, as previously reported $(27,28)$. In light of these prior reports, we here adopted HDM-sensitized mice as a representative mouse model of allergic skin inflammation. HDM, a mixture of various components including proteases and lipid-binding proteins, causes Th2 responses by its potential to activate TLR 4 signaling or its protease activity via DCs, epithelial cells, and basophils $(40,41)$. Furthermore, the mice epicutaneously sensitized or caused to inhale HDM showed phenotypes reminiscent of $\mathrm{AD}$ or asthma patients in this and other studies (Figure 1, Supplemental Figure 1, and refs. 27, 28, 41). The observations that chronic skin lesions of AD patients showed upregulation of IFN- $\gamma$ and IL-12 and that IFN- $\gamma$ deficiency partially improved ovalbumin-sensitized allergic skin inflammation in mice (42-44) are indicative of the involvement of Th1 responses in addition to Th2 responses. However, in our HDM-sensitized mouse model, enhancement of IFN- $\gamma$ expression in skin tissues was not observed, despite the appearance of other features of chronic $\mathrm{AD}$ skin lesions (Figure 1 and data not shown). Huang et al. have also reported that skin lesions in HDM-treated mice do not show IFN- $\gamma$ expression, whereas splenocytes responsive to allergen stimulation can induce IFN- $\gamma$ production (28). Exposure to microbial products may make the difference between mouse models and patients (4). Furthermore, Th17 responses have been implicated in the acute phase of $\mathrm{AD}$ pathogenesis, although this is still controversial $(4,45)$. Interestingly, DCs stimulated with KCM in the presence of IL-13 induced IL-17-producing T cells as well as IL-4/IL-13-producing T cells (Figure 2F). In spite of complicated and diverse phenotypes, our present study suggests that the induction of these phenotypes completely depended on STAT6, a critical transcriptional factor of Th2 cytokines (Figure 1 and Supplemental Figure 1). Furthermore, periostin induction may be a common feature in mouse models of allergic skin inflammation, because induction of periostin was observed not only in HDM-treated mice, but also in hapten-treated mice (Supplemental Figure 5).

Among the proinflammatory cytokines derived from activated $\mathrm{AD}$ keratinocytes, TSLP is critical in AD inflammation (26). TSLP 
A
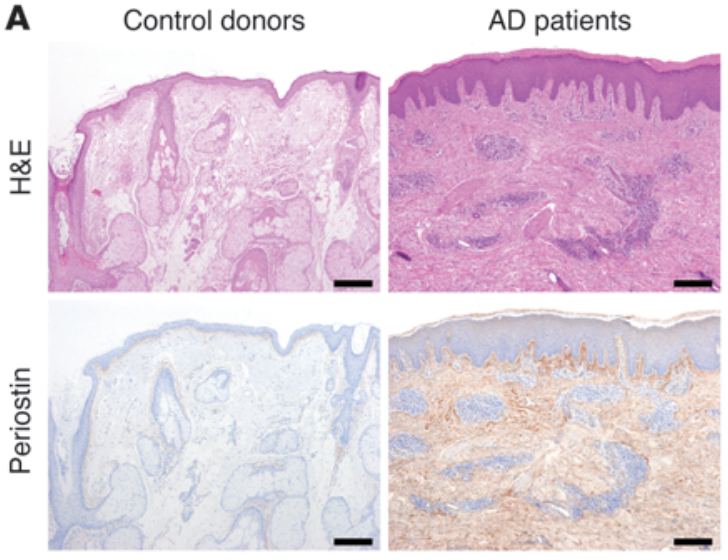

B
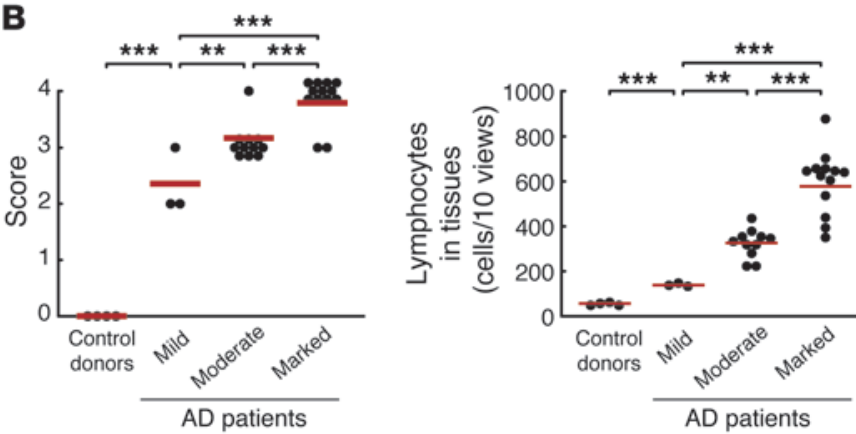

is produced mainly from epithelial cells together with other cells, such as mast cells, creating a Th2-permissive microenvironment via DCs $(26,46)$. Keratinocyte-specific overexpression of TSLP induces allergic skin inflammation in mice (47). Accordingly, production of TSLP is upregulated in keratinocytes derived from $\mathrm{AD}$ patients (46). In this study, we showed that KCM in the presence of periostin containing high amounts of TSLP induced Th2 skewing via DCs (Figure 2). Furthermore, recombinant periostin directly induced production of TSLP in keratinocytes (Figure 3). Addition of neutralizing Abs against TSLP into the IL-13-treated KCM partially inhibited Th2 skewing (Supplemental Figure 8). This result supports the involvement of TSLP in this event, although factors other than TSLP may also contribute to the Th2 skewing. Furthermore, the observation that neutralizing Abs against TSLP slightly downregulated Th2 skewing, even in the PBS-treated $\mathrm{KCM}$, indicated that basal production of periostin from untreated fibroblasts can cause induction of TSLP in keratinocytes, followed by Th2 skewing via DCs to a small extent. It has been reported that synergistic stimuli of Th2 cytokines (IL-4 or IL-13) and proinflammatory cytokines (TNF- $\alpha$ or IL-1 $\alpha$ ), or extrinsic stimuli via several TLRs, induce TSLP production in keratinocytes (48-50). In addition to these factors, we demonstrated that periostin was an intrinsic inducer of TSLP from keratinocytes, a finding we believe to be novel. It is known that signals via $\alpha_{v} \beta_{3}$ integrin lead to activation of NF- $\mathrm{KB}$, which is important for TSLP expression $(51,52)$. Indeed, TSLP production by periostin was dependent on $\alpha_{v} \beta_{3}$ integrin/NF- $\mathrm{KB}$ pathways (Figure 3 and Figure 4B). These results are consistent with a previous report that mice defective in $I \kappa B \alpha$, an inhibitor of the NF- $\mathrm{KB}$ pathway, develop inflammatory changes (53), showing the importance of NF-KB activation in keratinocytes in allergic skin inflammation.
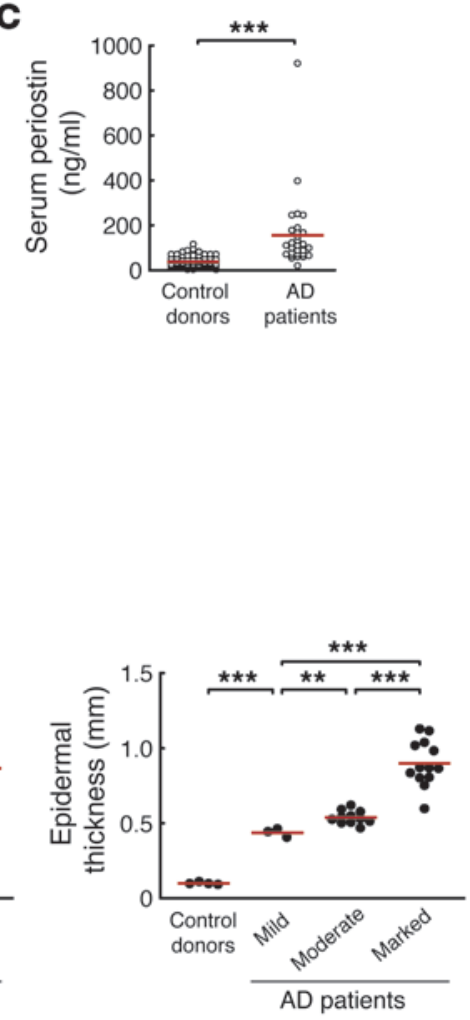

\section{Figure 6}

Periostin is highly expressed in skin tissues of AD patients, correlated with disease severity. (A) Skin tissues stained with $\mathrm{H} \& \mathrm{E}$ and anti-periostin $\mathrm{Ab}$ of a control donor and an AD patient with marked periostin expression. Scale bars: 200 $\mu \mathrm{m}$. (B) Inflammation score, lymphocyte number in the dermis, and epidermal thickness in healthy controls and AD patients with periostin expression classified as mild $(n=3)$, moderate $(n=11)$, or marked ( $n=13)$. Lymphocyte numbers were counted and epidermal thickness measured in $10 \times 400$ fields for each sample. (C) Periostin levels in sera obtained from control donors $(n=66)$ and AD patients $(n=29)$ using ELISA. Red lines in $\mathbf{B}$ and $\mathbf{C}$ indicate means. ${ }^{\star \star} P<0.01$, ${ }^{* * *} P<0.001$.
Hyperplasia and dysregulated differentiation of the epidermis (i.e., acanthosis) are typical features of chronic inflammatory skin diseases, including AD and psoriasis $(3,4,54)$. Several cytokines in addition to Th 2 cytokines - IL- $1 \alpha$, IL-21, IL-22, and IFN- $\gamma$ - are reported to be correlated with the generation of acanthosis (42, $45,55,56)$. In this study, we demonstrated that periostin acted as a critical player for acanthosis downstream of IL-4 or IL-13 signals. Furthermore, we showed that periostin induced activation of the Akt pathway through integrins (Supplemental Figure 11), and the Akt pathway is known to be strongly associated with keratinocyte survival and differentiation (34). Together with the NF-кB pathway, another important signal for proliferation of keratinocytes (57), the Akt pathway could be responsible for periostin-induced acanthosis. The pathophysiological role of acanthosis in chronic inflammation remains unknown. We demonstrated that periostinstimulated keratinocytes enhanced production of TSLP (Figure 3) and that production of proinflammatory cytokines was further enhanced by the increased number of activated keratinocytes (Figure 2). Overall, we assume that acanthosis in $\mathrm{AD}$ provides a basis for supplying more massive proinflammatory mediators from keratinocytes. Furthermore, based on the observation that Th2type responses functioned as an immune system against parasite infection, periostin may contribute to enforcement of this defense mechanism by activation of keratinocytes.

We showed that administration of neutralizing anti- $\alpha_{v}$ integrin Abs completely suppressed HDM-induced phenotypic changes (Figure 4). Because it is known that several ECM proteins - vitronectin, osteopontin, and thrombospondin - bind to $\alpha_{v}$ integrin (58), we cannot exclude the possibility that the effects of anti- $\alpha_{v}$ integrin Abs were the consequence of the inhibition of all functions of $\alpha_{v}$ integrin ligands, including periostin. However, we observed that TSLP 


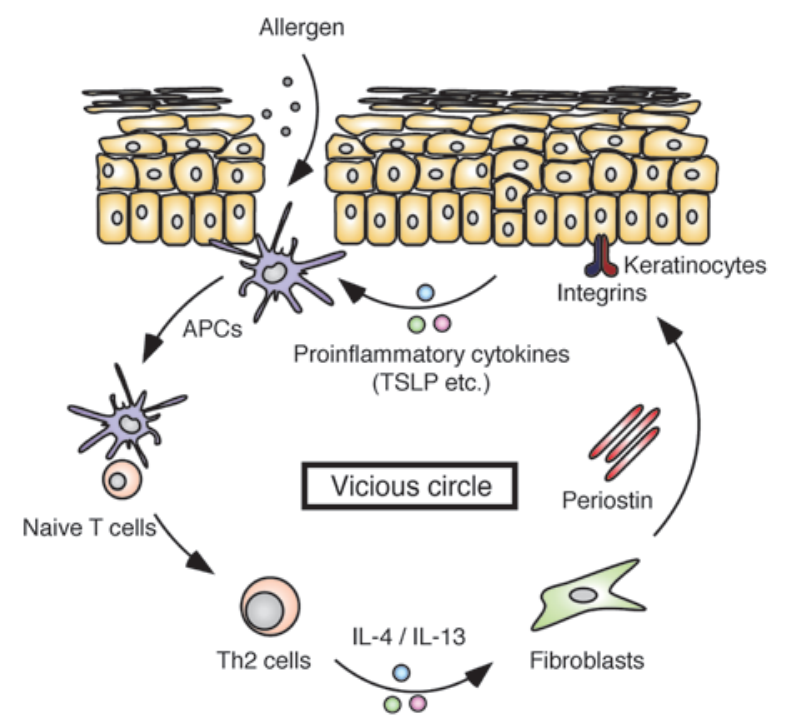

production was induced on periostin-coated, but not vitronectincoated, plates (data not shown). These results suggest that each of the $\alpha_{v}$ integrin ligands transduces different signals and that some receptors other than $\alpha_{v}$ integrin may be involved in these ligands.

It has been reported that allergen avoidance has limited efficacy $(59,60)$. Given that the intrinsic amplification pathway is important for chronicity of $\mathrm{AD}$, periostin and its functional receptor, $\alpha_{\mathrm{V}}$ integrin, can be a good target to develop therapeutic agents for AD. The blockage of $\alpha_{v}$ integrin was effective even in the HDM-sensitized mice (Figure 5). These results support our model and suggest that an agent blocking the interaction between periostin and $\alpha_{v}$ integrin might act as a not only preventive, but also therapeutic, drug. Antagonists against $\alpha_{v} \beta_{3}$ integrin have been tested in clinical use for tumors, rheumatoid arthritis, and osteoporosis (61-63). However, it is assumed that blockage of periostin has an advantage compared with blockage of $\alpha_{v}$ integrin, because it has been shown that most $\alpha_{v}$ integrin-deficient mice die before birth (64), so that blockage of $\alpha_{v}$ integrin will give more severe adverse effects than blockage of periostin. However, we must be careful concerning the possible risk of adverse effects to block the action of periostin on cardiac development/remodeling or cutaneous wound repair, because periostin plays important roles in these events $(11,12$, 15-17). The present findings provide a molecular basis for considering periostin as a potential therapeutic target for AD in humans.

\section{Methods}

Mice. 8-week-old BALB/c mice, C57BL/6 mice, NC/Nga mice (all Japan SLC), and Stat $6^{-/}$mice (gift from T. Hoshino, Kurume University School of Medicine, Kurume, Japan) were used. Postn ${ }^{-/}$mice, prepared as previously described (129SJv;C57BL/6 background; ref. 11), were backcrossed to BALB/c mice, and F4-F8 mice were used. Postn ${ }^{-1-}$ mice were always evaluated together with their WT littermates.

HDM preparation. Dermatophagoides farinae (gift of Torii Pharmaceutical) was defatted with ethyl ether, then extracted in 50\% glycerin and 5\% $\mathrm{NaCl}$ buffer at $4^{\circ} \mathrm{C}$ for 48 hours under constant stirring. After filtration, D. farinae extract was used as HDM.

Allergic skin inflammation model. The mouse model of allergic skin inflammation was generated as previously described (27), with minor modifications. Both surfaces of the ear lobes were stripped 3 times using cellophane

\section{Figure 7}

Vicious circle in allergic skin inflammation, composed of IL-4/IL-13, periostin, and proinflammatory cytokines from keratinocytes. The exposure of APCs, including DCs, to allergens induces Th2-type responses. IL-4 and IL-13 derived from Th2 cells stimulate fibroblasts to produce periostin. Periostin binds to $\alpha_{v}$ integrin on keratinocytes, inducing proinflammatory cytokines, including TSLP. The proinflammatory cytokines derived from keratinocytes enhance Th2 inflammation. tape (Kyowa), and $25-\mu \mathrm{l}$ HDM $(10 \mathrm{mg} / \mathrm{ml})$ solution was painted onto both surfaces of the earlobes. This procedure was performed once per week for 7 weeks. Ear swelling was measured before each HDM painting with a micrometer (Ozaki MFG). The mouse ear tissues, cervical lymph nodes, and serum were collected 24 hours after the last HDM painting. $10 \mu \mathrm{l}$ anti- $\alpha_{v}$ integrin $\mathrm{Ab}(1 \mathrm{mg} / \mathrm{ml}$; BioLegend) or rat IgG1 isotype control $\mathrm{Ab}$ (1 mg/ $\mathrm{ml}$; R\&D Systems) was administered subcutaneously to ear tissues 6 hours before and 3 days after all HDM paintings (prevention protocol), or from the fourth HDM painting until day 21 (therapeutic protocol).

Histology. Ear tissues were subjected to histological staining with $\mathrm{H} \& \mathrm{E}$, Masson trichrome, and toluidine blue. Immunohistochemical staining was performed as previously described (19); see Supplemental Methods for Abs. Apoptotic cells were detected by TUNEL assay (Promega). Epidermal thickness was measured, and the number of lymphocytes, eosinophils, and mast cells counted, in $10 \times 400$ fields for each mouse or human sample, and the averages values were calculated.

IgE. Duplicate serum samples were analyzed using an ELISA kit for total IgE (Bethyl Laboratories).

Cells. See Supplemental Methods for preparation of mouse keratinocytes and dermal fibroblasts. Mouse embryonic fibroblasts were obtained from E14.5 embryos of Postn ${ }^{+/-}$or Postn ${ }^{-/-}$mice. For stimulation, either human or mouse dermal fibroblasts were stimulated by $10-50 \mathrm{ng} / \mathrm{ml} \mathrm{IL-4}$ or IL-13 (PeproTech).

RT-PCR. RT-PCR for Postn and Gapdh was performed as previously described (19). See Supplemental Methods for details.

Western blotting. Western blotting for periostin and GAPDH was performed as previously described (19).

3-dimensional organotypic coculture of keratinocytes with fibroblasts. Mouse keratinocytes were cultured using a 3-dimensional system (Figure 3A) modified from a previously reported system (33). $2 \mathrm{ml}$ type I collagen gel solution (Nitta Gelatin) containing $2 \times 10^{5}$ mouse embryonic fibroblasts was poured into an inner dish with a bottom made of nitrocellulose membrane (Millicell-CM). Next, $2 \times 10^{6}$ keratinocytes in total were overlaid on the gel, and DMEM was poured into the outer dish with the surface level lower than that of the gel, to let the keratinocytes face the air. The culture medium was replaced every 2 days. In some experiments, $10 \mathrm{ng} / \mathrm{ml}$ mouse IL-13, $10 \mu \mathrm{g} /$ $\mathrm{ml}$ anti- $\alpha_{\mathrm{v}}$ integrin $\mathrm{Ab}$, or $10 \mu \mathrm{g} / \mathrm{ml}$ anti- $\beta_{3}$ integrin $\mathrm{Ab}$ (BD Biosciences) was added. In some experiments, the NF- $\mathrm{KB}$ inhibitor BAY 11-7082 (concentrations as indicated; Sigma-Aldrich) was added into the medium. 
Recombinant proteins. The recombinant protein coded by $\mathrm{pMT} / \mathrm{Bip} /$ V5-HisA-periostin was prepared as previously described (19).

Quantitative analysis of cytokines. Amounts of TSLP, TNF- $\alpha$, and GM-CSF in the culture medium were quantified with ELISA kits for mouse TSLP (R\&D Systems), TNF- $\alpha$, and GM-CSF (eBioscience).

Mixed lymphocyte reaction. Bone marrow-derived DCs were prepared from a bone marrow suspension obtained from the femurs and tibias of C57BL/ 6 mice. Cells were cultured with $20 \mathrm{ng} / \mathrm{ml}$ murine GM-CSF (PeproTech) for 6 days. CD4 $4^{+} \mathrm{T}$ cells were prepared from spleen and lymph node cells of BALB/c mice using anti-CD4-coated MACS magnetic beads (Miltenyi Biotec, Germany). In total, $2 \times 10^{5} \mathrm{CD}^{+} \mathrm{T}$ cells were cocultured with KCM-treated bone marrow-derived DCs. Cells were harvested after 3 or 5 days for $\left[{ }^{3} \mathrm{H}\right]$ thymidine incorporation assay or quantitative RT-PCR analysis, respectively. Quantitative RT-PCR analysis was performed after restimulation of the cells with anti-CD3 Ab and anti-CD28 Ab for 24 hours.

Confocal microscopy. Keratinocytes were fixed by paraformaldehyde. After blocking with $2 \%$ BSA, sections were stained by anti-NF- $\mathrm{B}$ p 65 Ab (Santa Cruz Biotechnology), followed by Alexa Fluor 488-labeled anti-rabbit IgG $\mathrm{Ab}$ (Invitrogen), or TO-PRO-3 (Invitrogen) for the nucleus. Sections were mounted by Dako Fluorescent Mounting Medium (Dako) and then examined by LSM5 PASCAL G/B (Carl Zeiss).

Human skin tissue specimens. Skin tissues examined for histology were derived from 4 normal donors and $27 \mathrm{AD}$ patients, and serum samples were derived from 66 normal donors and $29 \mathrm{AD}$ patients. The $27 \mathrm{AD}$ patients for histology were aged 13-54 years; 2 had received oral prednisolone, and 19 had received topical steroid ointments. The $29 \mathrm{AD}$ patients for serum were aged $13-53$ years; 3 had received oral prednisolone, and 27 had received topical steroid ointments (1 patient had received both). Patients received steroid treatment at the time of sample collection. No $\mathrm{AD}$ patient had bronchial asthma or rhinosinusitis. We scored the severity of skin lesions on a $0-4$ scale according to the guidelines established by the Japanese Dermatological Association (65). We grouped the patient sample into 3 types, according to the degree and pattern of periostin expression: mild (mild expression on the border of the epidermis), moderate (strong expression on the border of the epidermis), and marked (strong and broad increase in the dermis).

Measurement of serum periostin. We performed a human periostin ELISA assay using 2 rat anti-human periostin mAbs (clones no. SS18A and SS17B), as previously reported (66). See Supplemental Methods for details.

Statistics. Results are presented as means + SD in all experiments. Analysis was carried out using 2-sided, unpaired Student's $t$ test or 2-sided Welch test. A $P$ value less than 0.05 was considered significant.

Study approval. Animal experiments were undertaken following the guidelines for care and use of experimental animals of the Japanese Association for Laboratory Animals Science (1987) and were approved by the Saga University Animal Care and Use Committee (Saga, Japan). Human study protocols were approved by the Ethics Committees of Kyushu University and Saga Medical School. Informed consent was obtained from all patients and healthy volunteers.

\section{Acknowledgments}

We thank Koichi Akashi, Dovie R. Wylie, Hiroyuki Ideguchi, and Yumiko Ohishi for critical review of this manuscript and technical assistance. This work was supported in part by Grants-in-Aid for Scientific Research from the Japan Society for the Promotion of Science.

Received for publication May 12, 2011, and accepted in revised form May 2, 2012.

Address correspondence to: Kenji Izuhara, Division of Medical Biochemistry, Department of Biomolecular Sciences, Saga Medical School, 5-1-1, Nabeshima, Saga 849-8501, Japan. Phone: 81.952. 34.2261; Fax: 81.952.34.2058; E-mail: kizuhara@cc.saga-u.ac.jp.
1. Medzhitov R. Inflammation 2010: new adventures of an old flame. Cell. 2010;140(6):771-776.

2. Kaiko GE, Foster PS. New insights into the generation of Th2 immunity and potential therapeutic targets for the treatment of asthma. Curr Opin Allergy Clin Immunol. 2011;11(1):39-45.

3. Leung DY, Boguniewicz M, Howell MD, Nomura I, Hamid QA. New insights into atopic dermatitis. J Clin Invest. 2004;113(5):651-657.

4. Oyoshi MK, He R, Kumar L, Yoon J, Geha RS. Cellular and molecular mechanisms in atopic dermatitis. Adv Immunol. 2009;102:135-226.

5. Meneghin A, Hogaboam CM. Infectious disease, the innate immune response, and fibrosis. $J$ Clin Invest. 2007;117(3):530-538.

6. Wynn TA. Fibrotic disease and the $\mathrm{T}_{\mathrm{H}} 1 / \mathrm{T}_{\mathrm{H}} 2$ paradigm. Nat Rev Immunol. 2004;4(8):583-594.

7. Midwood $\mathrm{K}$, et al. Tenascin- $\mathrm{C}$ is an endogenous activator of Toll-like receptor 4 that is essential for maintaining inflammation in arthritic joint disease. Nat Med. 2009;15(7):774-780.

8. Shinohara ML, Kim JH, Garcia VA, Cantor H. Engagement of the type I interferon receptor on dendritic cells inhibits Thelper 17 cell development: role of intracellular osteopontin. Immunity. 2008; 29(1):68-78.

9. Hamilton DW. Functional role of periostin in development and wound repair: implications for connective tissue disease. J Cell Commun Signal. 2008; 2(1-2):9-17.

10. Ruan K, Bao S, Ouyang G. The multifaceted role of periostin in tumorigenesis. Cell Mol Life Sci. 2009; 66(14):2219-2230.

11. Rios $\mathrm{H}$, et al. periostin null mice exhibit dwarfism, incisor enamel defects, and an early-onset peri- odontal disease-like phenotype. Mol Cell Biol. 2005; 25(24):11131-11144.

12. Snider $P$, et al. Periostin is required for maturation and extracellular matrix stabilization of noncardiomyocyte lineages of the heart. Circ Res. 2008; 102(7):752-760.

13. Kühn B, et al. Periostin induces proliferation of differentiated cardiomyocytes and promotes cardiac repair. Nat Med. 2007;13(8):962-969.

14. Shimazaki M, et al. Periostin is essential for cardiac healing after acute myocardial infarction. $J$ Exp Med. 2008;205(2):295-303.

15. Nishiyama T, et al. Delayed re-epithelialization in periostin-deficient mice during cutaneous wound healing. PLoS One. 2011;6(4):e18410.

16. Elliott CG, et al. Periostin modulates myofibroblast differentiation during full-thickness cutaneous wound repair. J Cell Sci. 2012;125(pt 1):121-132.

17. Ontsuka K, et al. Periostin, a matricellular protein, accelerates cutaneous wound repair by activating dermal fibroblasts. Exp Dermatol. 2012;21(5):331-336.

18. Hayashi N, Yoshimoto T, Izuhara K, Matsui K, Tanaka T, Nakanishi K. T helper 1 cells stimulated with ovalbumin and IL-18 induce airway hyperresponsiveness and lung fibrosis by IFN- $\gamma$ and IL-13 production. Proc Natl Acad Sci U S A. 2007; 104(37):14765-14770.

19. Takayama G, et al. Periostin: a novel component of subepithelial fibrosis of bronchial asthma downstream of IL-4 and IL-13 signals. J Allergy Clin Immunol. 2006;118(1):98-104.

20. Kii I, et al. Incorporation of tenascin-C into the extracellular matrix by periostin underlies an extracellular meshwork architecture. J Biol Chem. 2010; 285(3):2028-2039.
21. Maruhashi T, Kii I, Saito M, Kudo A. Interaction between periostin and BMP-1 promotes proteolytic activation of lysyl oxidase. J Biol Chem. 2010; 285(17):13294-13303.

22. Uchida $M$, et al. Periostin, a matricellular protein, plays a role in the induction of chemokines in pulmonary fibrosis. Am J Respir Cell Mol Biol. 2012;46(5):677-686.

23. Blanchard C, et al. Periostin facilitates eosinophil tissue infiltration in allergic lung and esophageal responses. Mucosal Immunol. 2008;1(4):289-296.

24. Sidhu SS, et al. Roles of epithelial cell-derived periostin in TGF- $\beta$ activation, collagen production, and collagen gel elasticity in asthma. Proc Natl Acad Sci US A. 2010;107(32):14170-14175.

25. Carmi-Levy I, Homey B, Soumelis V. A modular view of cytokine networks in atopic dermatitis. Clin Rev Allergy Immunol. 2011;41(3):245-253.

26. Liu YJ, et al. TSLP: an epithelial cell cytokine that regulates $\mathrm{T}$ cell differentiation by conditioning dendritic cell maturation. Annu Rev Immunol. 2007;25:193-219.

27. Gao XK, Nakamura N, Fuseda K, Tanaka H, Inagaki N, Nagai H. Establishment of allergic dermatitis in NC/Nga mice as a model for severe atopic dermatitis. Biol Pharm Bull. 2004;27(9):1376-1381.

28. Huang CH, Kuo IC, Xu H, Lee YS, Chua KY. Mite allergen induces allergic dermatitis with concomitant neurogenic inflammation in mouse. J Invest Dermatol. 2003;121(2):289-293.

29. Jensen JM, et al. Impaired sphingomyelinase activity and epidermal differentiation in atopic dermatitis. J Invest Dermatol. 2004;122(6):1423-1431.

30. Howell MD, et al. Cytokine modulation of atopic dermatitis filaggrin skin expression. J Allergy Clin 
Immunol. 2007;120(1):150-155

31. Izuhara K, Arima K, Kanaji S, Ohta S, Kanaji T. IL-13: a promising therapeutic target for bronchial asthma. Curr Med Chem. 2006;13(19):2291-2298.

32. Yuyama N, et al. Analysis of novel disease-related genes in bronchial asthma. Cytokine. 2002; 19(6):287-296

33. Aoki S, Toda S, Ando T, Sugihara H. Bone marrow stromal cells, preadipocytes, and dermal fibroblasts promote epidermal regeneration in their distinctive fashions. Mol Biol Cell. 2004;15(10):4647-4657.

34. Muller EJ, Williamson L, Kolly C, Suter MM. Outsidein signaling through integrins and cadherins: a central mechanism to control epidermal growth and differentiation? J Invest Dermatol. 2008;128(3):501-516.

35. Griffiths CE, Dearman RJ, Cumberbatch M, Kimber I. Cytokines and Langerhans cell mobilisation in mouse and man. Cytokine. 2005;32(2):67-70.

36. Zou GM, Tam YK. Cytokines in the generation and maturation of dendritic cells: recent advances. Eur Cytokine Netw. 2002;13(2):186-199.

37. Norris RA, Moreno-Rodriguez R, Hoffman S, Markwald RR. The many facets of the matricelluar protein periostin during cardiac development, remodeling, and pathophysiology. J Cell Commun Signal. 2009;3(3-4):275-286

38. Sehra S, et al. Periostin regulates goblet cell metaplasia in a model of allergic airway inflammation. J Immunol. 2011;186(8):4959-4966.

39. Gordon ED, et al. A protective role for periostin and TGF- $\beta$ in IgE-mediated allergy and airway hyperresponsiveness. Clin Exp Allergy. 2012;42(1):144-155.

40. Arlian LG, Platts-Mills TA. The biology of dust mites and the remediation of mite allergens in allergic disease. J Allergy Clin Immunol. 2001; 107(3 suppl):S406-S413.

41. Hammad H, et al. Inflammatory dendritic cellsnot basophils-are necessary and sufficient for induction of Th2 immunity to inhaled house dust mite allergen. J Exp Med. 2010;207(10):2097-2111.

42. Spergel JM, Mizoguchi E, Oettgen H, Bhan AK, Geha RS. Roles of TH1 and TH2 cytokines in a murine model of allergic dermatitis. J Clin Invest. 1999;103(8):1103-1111.

43. Grewe M, Gyufko K, Schopf E, Krutmann J. Lesional expression of interferon-gamma in atopic eczema. Lancet. 1994;343(8888):25-26.

44. Hamid Q, Naseer T, Minshall EM, Song YL, Boguniewicz M, Leung DY. In vivo expression of IL-12 and IL-13 in atopic dermatitis. J Allergy Clin Immunol. 1996;98(1):225-231.

45. Souwer Y, Szegedi K, Kapsenberg ML, de Jong EC. IL-17 and IL-22 in atopic allergic disease. Curr Opin Immunol. 2010;22(6):821-826.

46. Soumelis V, et al. Human epithelial cells trigger dendritic cell mediated allergic inflammation by producing TSLP. Nat Immunol. 2002;3(7):673-680.

47. Yoo J, et al. Spontaneous atopic dermatitis in mice expressing an inducible thymic stromal lymphopoietin transgene specifically in the skin. J Exp Med. 2005;202(4):541-549.

48. Bogiatzi SI, et al. Proinflammatory and Th2 cytokines synergize to induce thymic stromal lymphopoietin production by human skin keratinocytes. J Immunol. 2007;178(6):3373-3377.

49. Kinoshita H, et al. Cytokine milieu modulates release of thymic stromal lymphopoietin from human keratinocytes stimulated with doublestranded RNA. J Allergy Clin Immunol. 2009; 123(1):179-186

50. Vu AT, et al. Staphylococcus aureus membrane and diacylated lipopeptide induce thymic stromal lymphopoietin in keratinocytes through the Toll-like receptor 2-Toll-like receptor 6 pathway. J Allergy Clin Immunol. 2010;126(5):985-993

51. Lee HC, Ziegler SF. Inducible expression of the proallergic cytokine thymic stromal lymphopoietin in airway epithelial cells is controlled by NFKB. Proc Natl Acad Sci U S A. 2007;104(3):914-919.

52. Scatena M, Almeida M, Chaisson ML, Fausto N, Nicosia RF, Giachelli CM. NF-kB mediates $\alpha_{v} \beta_{3}$ integrin-induced endothelial cell survival. $J$ Cell Biol. 1998;141(4):1083-1093.

53. Rebholz B, et al. Crosstalk between keratinocytes and adaptive immune cells in an IKB $\alpha$ proteinmediated inflammatory disease of the skin. Immunity. 2007;27(2):296-307.

54. Korn T, Bettelli E, Oukka M, Kuchroo VK. IL-17 and Th17 cells. Annu Rev Immunol. 2009;27:485-517.

55. Caruso R, et al. Involvement of interleukin- 21 in the epidermal hyperplasia of psoriasis. Nat Med. 2009; 15(9):1013-1015.

56. Szabowski A, et al. c-Jun and JunB antagonistically control cytokine-regulated mesenchymal-epidermal interaction in skin. Cell. 2000;103(5):745-755.

57. Karin M. Nuclear factor- $\mathrm{\kappa B}$ in cancer development and progression. Nature. 2006;441(7092):431-436.

58. Clemmons DR, Maile LA, Ling Y, Yarber J, Busby WH. Role of the integrin $\alpha_{v} \beta_{3}$ in mediating increased smooth muscle cell responsiveness to IGF-I in response to hyperglycemic stress. Growth Horm IGF Res. 2007;17(4):265-270.

59. Romei I, Boner AL. Possible reasons for lack of effect of allergen avoidance in atopy-prone infants and sensitive asthmatic patients. Clin Rev Allergy Immunol. 2005;28(1):59-71.

60. Simpson EL. Atopic dermatitis prevention. Dermatol Ther. 2006;19(2):108-117.

61. Cacciari B, Spalluto G. Non peptidic $\alpha_{v} \beta_{3}$ antagonists: recent developments. Curr Med Chem. 2005; 12(1):51-70.

62. Jin H, Varner J. Integrins: roles in cancer development and as treatment targets. Br J Cancer. 2004; 90(3):561-565.

63. Lainer-Carr D, Brahn E. Angiogenesis inhibition as a therapeutic approach for inflammatory synovitis. Nat Clin Pract Rheumatol. 2007;3(8):434-442.

64. Bader BL, Rayburn H, Crowley D, Hynes RO. Extensive vasculogenesis, angiogenesis, and organogenesis precede lethality in mice lacking all $\alpha \mathrm{v}$ integrins. Cell. 1998;95(4):507-519.

65. Furue M, et al. Guidelines for management of atopic dermatitis. Jpn J Dermatol. 2008;118:325-342.

66. Okamoto M, et al. Periostin, a matrix protein, is a novel biomarker for idiopathic interstitial pneumonias. Eur Respir J. 2011;37(5):1119-1127. 